\title{
The method of reflow and online electrolysis in vanadium redox battery: benefits and limitations
}

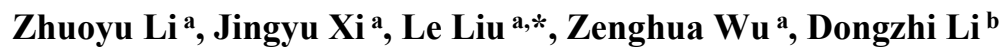

${ }^{a}$ Institute of Green Chemistry and Energy, Tsinghua Shenzhen International Graduate School, Tsinghua University, Shenzhen 518055, China

b Department of Physics, School of Science, Shenyang University of Technology, Shenyang, China.

*Corresponding authors

Le Liu (Email: liu.le@sz.tsinghua.edu.cn Tel: +8675526036181)

Authors

Zhuoyu Li (Email: lizhuoyu17@mails.tsinghua.edu.cn )

Jingyu Xi (Email: xijy@sz.tsinghua.edu.cn )

Zenghua Wu (Email: wuzh@mail.tsinghua.edu.cn )

Dongzhi Li (Email: lidongzhi@tsinghua.org.cn ) 


\section{TABLE OF CONTENTS}

Reflow versus Reflow+Electrolysis (this work) S3

Remixing versus Remixing+Electrolysis $\quad$ S3-S4

Rimixing versus Reflow+Electrolysis (this work) $\quad$ S4

Rimixing+Electrolysis versus Reflow+Electrolysis (this work) S4-S5

Figure S1. The capacity retentions of VFBs with reflow and reflow with online electrolysis

S3

Figure S2. The capacity retentions of VFBs with remixing and the method of remixing and electrolysis

Figure S3. The capacity retentions of VFBs with remixing and the method of reflow and electrolysis in this work

3 Figures

4 Pages 
Our group have proposed a few methods to handle the capacity decay problem in VFBs (including this work). In this Supporting Information, the results of these methods are compared to show the advantage of the method proposed in this work.

\section{Reflow versus Reflow+Electrolysis (this work)}

Our group has proposed many solutions to alleviate capacity imbalance. In 2017, we have proposed a novel electrolyte-reflow method to reduce capacity decay and prolong cycle life of VFBs (J. Power Sources, 338 (2017) 17-25, Ref. 32 in the manuscript). However, the concentration of $\mathrm{VO}_{2}{ }^{+}$in each reflowed VFB is rather higher than regular VFB, which leads to the acceleration of the discharge capacity decay rate and cause a danger of vanadium precipitation. Hence, in this work, we combine the method of online electrolysis with the reflow method to reduce the accumulated $\mathrm{VO}_{2}{ }^{+}$ions in the positive electrolyte and restore the capacity. And the results showed that the capacity is restored apparently and the VFB runs 4680 cycles stably through eight online electrolysis. As shown in Figure S1, comparing with the reflow method, the method of reflow with online electrolysis in this work effectively prolongs the cycle life, from 954 to 4680 cycles.

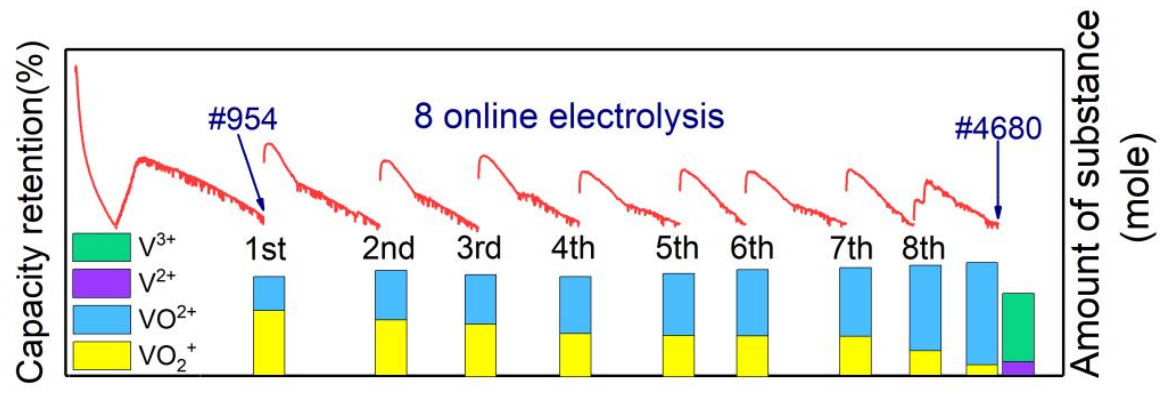

Figure S1. The capacity retentions of VFBs with reflow and reflow with online electrolysis

\section{Remixing versus Remixing+Electrolysis}

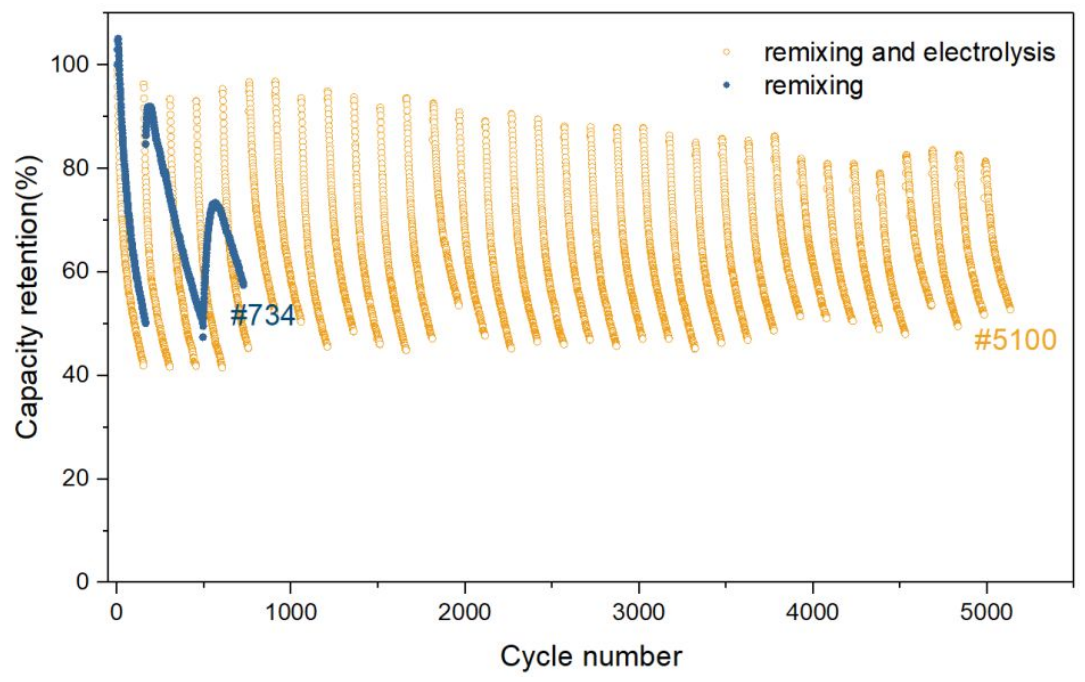

Figure S2. The capacity retentions of VFBs with remixing (Appl. Energy, 204 (2017) 373-381, Ref. 33 in the revised manuscript) and the method of remixing and electrolysis (J. Power Sources, 438 (2019) 226990, Ref. 38 in the manuscript) 
In 2018, we have explored the benefits and limitations of the remixing method, and found that the contribution of the remixing method is restrained by the rising average valence of the mixed electrolytes (Appl. Energy, 204 (2017) 373-381, Ref. 33 in the manuscript). Hence, we added online electrolysis to the remixing method, and this part of the work was published in 2019 (J. Power Sources, 438 (2019) 226990, Ref. 38 in the manuscript). The results showed that through remixing and online electrolysis, after 5100 charge-discharge cycles, the vanadium battery can still maintain a capacity retention of more than $80 \%$. As shown in Figure S2, comparing with the remixing method, the method of remixing and online electrolysis effectively prolongs the cycle life, from 734 to 5100 cycles.

\section{Rimixing versus Reflow+Electrolysis (this work)}

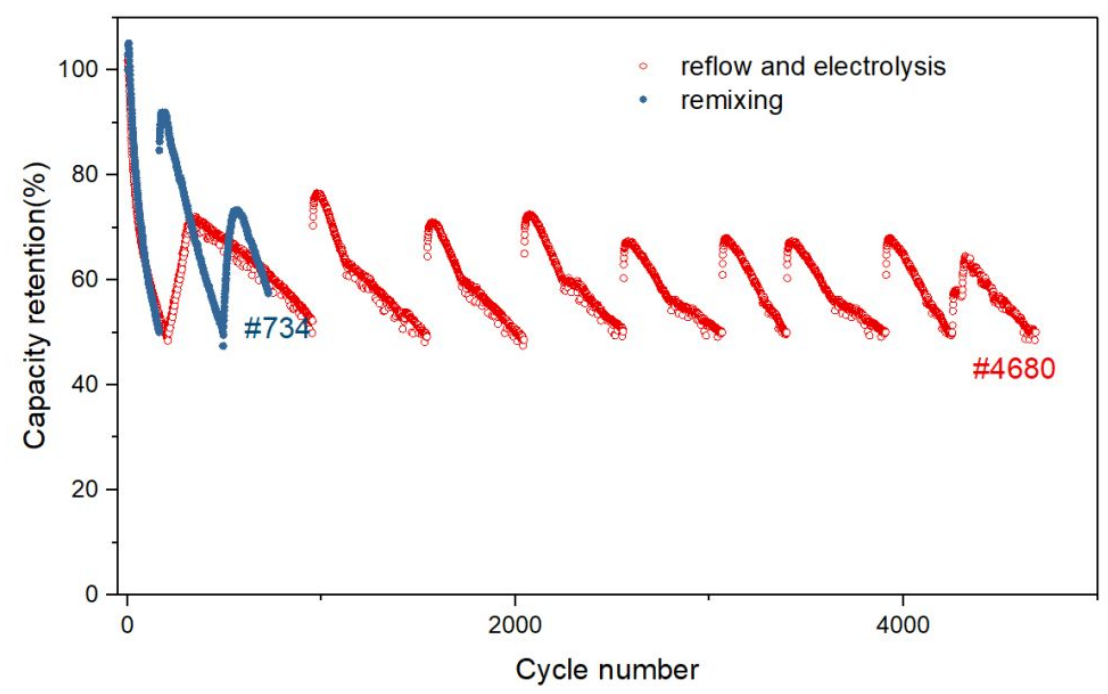

Figure S3. The capacity retentions of VFBs with remixing (Appl. Energy, 204 (2017) 373-381, Ref. 33 in the revised manuscript) and the method of reflow and electrolysis in this work

Fig. S3 compared the capacity retention of the VFB with remixing (Appl. Energy, 204 (2017) 373-381, Ref. 33 in the manuscript) and reflow with online electrolysis in this work. As shown in Figure S3, the recovery effect of the remixing method is getting worse, and became invalid after two remixing processes. The cycle number is increased from 145 to 734 by remixing at the current density of $160 \mathrm{~mA} \mathrm{~cm}^{-2}$, which is far below the 4680 cycles by the reflow and electrolysis method in this work.

\section{Rimixing+Electrolysis versus Reflow+Electrolysis (this work)}

Compared the capacity retention of the VFB with the remixing and online electrolysis $(J$. Power Sources, 438 (2019) 226990, Ref. 38 in the manuscript) in Figure S2 with the one with reflow and online electrolysis in Figure S3 in this work, we can find that the method of remixing and online electrolysis have more cycle numbers (5100) than this work (4680). However, the reflow with online electrolysis method in this work have its unique advantages:

(1) It doesn't need to remix the positive and negative electrolyte, saving a lot of time and energy for large scale energy storage system which has thousands of tons of electrolytes.

(2) It doesn't need a pre-charge process after each electrolysis, which also save a lot of time 
and energy for large scale energy storage system with MWh scaled capacity.

(3) It has lower maintenance frequency: It only need $\mathbf{8}$ online electrolysis processes during 4680 cycles, while the method of remixing and electrolysis need 33 online electrolysis processes during 5100 cycles.

To sum up, the reflow with online electrolysis method in this work is more suitable to be applied in large scale VFB energy storage systems. 